\title{
Inference of Online Auction Shills Using Dempster-Shafer Theory*
}

\author{
Fei Dong ${ }^{1}$, Sol M. Shatz ${ }^{1}$, and Haiping $\mathrm{Xu}^{2}$ \\ ${ }^{1}$ Department of Computer Science \\ University of Illinois at Chicago, Chicago, IL 60607, USA \\ E-mail: \{fdong3, shatz\}@uic.edu \\ ${ }^{2}$ Computer and Information Science Department \\ University of Massachusetts Dartmouth, North Dartmouth, MA 02747, USA \\ E-mail:hxu@umassd.edu
}

\begin{abstract}
We present a shilling behavior detection and verification approach for online auction systems. Assuming a model checking technique to detect shill suspects in real-time, we focus on how to verify shill suspects using Dempster-Shafer theory of evidence. To demonstrate the feasibility of our approach, we provide a case study using real eBay auction data. The analysis results show that our approach can detect shills and that using Dempster-Shafer theory to combine multiple sources of evidence of shilling behavior can reduce the number of false positive results that would be generated from a single source of evidence.
\end{abstract}

\section{Introduction}

Online auction business grows when e-commerce becomes increasingly popular. For example, eBay, the world's largest auction website, announced \$2.19 billion revenue for the first quarter of 2008. However, because online auction users do not deal with each other face to face, this contemporary business medium faces an important challenge - auction frauds, which are illegal activities in online auctions. Shill bidding is one of the most prevalent forms of auction fraud. A shill is a person who poses as a legitimate buyer and feigns enthusiasm for an auctioned item by bidding up the price; thus the shill can serve as a decoy to attract bids from legitimate users.

Shill bidding can cause online auction participants much monetary loss, and worst of all, it may not even

\footnotetext{
${ }^{*}$ This material is based upon work supported by the U.S. National Science Foundation under grant numbers CNS-0715648 and CNS0715657.
}

be noticed by the victims. Many shill bidding strategies or patterns have been identified and summarized to help investigate auction fraud $[1,4]$. Unfortunately, a large amount of the acquired knowledge involves uncertainty. For example, by investigating real auction data, we might form the following shill-bidding pattern: "when a bidder tends to place bids in an auction at a higher bidding price rather than in another concurrent auction with an identical auctioned item that has a lower bidding price, the bidder might be a shill." However, an explanation to this bidding pattern could also be that some experienced buyers may prefer highly rated sellers over low rated ones, even though the high rating sellers may sell the same item at a higher price. This is reasonable because in most scenarios, high reputation sellers are trusted for quality of service. Therefore, the match of a single bidding pattern is not recommended as obvious evidence of shills.

To detect shilling behavior accurately, we propose a two-step approach. In the first step, we adopt a model checking method to detect suspicious shilling behaviors. The details of this step have been presented in [1], which, to the best of our knowledge, is the first approach that may potentially support detection of shill suspects in real-time. To verify the detection results from the first step and to reduce the number of possible false positives, in the second step, we combine knowledge obtained in the first step - the combination is carried out using the mathematical theory of evidence, Dempster-Shafter (D-S) theory. This two-step process for shill inference outputs a shilling score that can assist an auction house with trust judgment for each shill suspect.

The paper is organized as follows. Related work is reviewed in Section 2. Section 3 presents our two-step approach: detecting potential shills using model checking techniques and then verifying a suspect's 
status based on Dempster-Shafer theory. A case study and analysis results are presented in Section 4. Section 5 concludes the paper and mentions future work.

\section{Related work}

Trust management in online auction systems. As part of our previous work, $\mathrm{Xu}$ et al. presented a multiagent trust management (ATM) framework for online auctions [2]. The shill inference procedure discussed in this paper can be embedded in the security agent of ATM. Xu et al. introduced a formal model checking approach to detect shilling behaviors, especially the competitive shilling behaviors [1]. Kauffman et al. statistically analyzed data from rare coin auctions on eBay, and empirically tested the questionable bidding behaviors that are attributable to shill bidding [3]. Trevathan et al. designed an algorithm based on pattern matching to detect shilling behaviors in online English auctions [4]. Chau et al. applied data mining and trust propagation techniques to detect fraudulent users in online auction systems [5].

Generally, these techniques suffer from two drawbacks. Data mining related approaches need to deal with a large amount of historical data; thus they may have limited value in detecting shill bidding in a time-efficient manner. Pattern matching based and model-checking based approaches do not regularly update prior knowledge with the presence of new evidence. Therefore they may frequently generate false positive results. In contrast, our proposed approach can not only detect suspicious shilling behavior efficiently, but can also make the results more accurate for online auctions.

Dempster-Shafer Theory. Information related to decision making is often uncertain and incomplete. Hence it is of vital importance to find a feasible way to make decisions under this uncertainty. D-S theory [8], a probabilistic reasoning technique, is designed to deal with uncertainty and incompleteness of available information. It is a powerful tool for combining accumulative evidence and changing prior knowledge in the presence of new evidence. D-S theory has been used widely for fraud detection and system verification. For example, Chen et al. presented a D-S based intrusion detection approach for $\mathrm{Ad} \mathrm{Hoc}$ networks [6]. In their system, data from multiple processors are combined to form a decision about a node's true identity. Panigrahi et al. used D-S theory to combine evidence to estimate the likelihood of fraud in the context of mobile phone fraud detection [7]. They demonstrated the effectiveness of D-S theory for their applications.
In this paper, we propose a unique shill verification method based on D-S theory. Our proposed methods can be used to combine multiple pieces of evidence with new evidence to categorize a shill suspect's genuineness, and thereafter to update the suspect's status in a complex shill detection system.

\section{Inference of shill bidding}

\subsection{An abstract model}

Our approach can be abstracted as a 5-tuple $<\mathrm{B}, \delta$, P, $\theta, \varphi>$, where

1. $\mathbf{B}=\left\{b_{1}, b_{2}, \ldots, b_{n}\right\}$ is a set of online auction bidders;

2. $\boldsymbol{\delta}: \mathrm{B} \rightarrow[0,1]$ is a scoring function. Every online auction bidder has a shilling score, and $\delta\left(b_{i}\right)$ is defined as the shilling score for bidder $i$;

3. $\mathbf{P}=\left\{\mathrm{p}_{1}, \mathrm{p}_{2}, \ldots, \mathrm{p}_{\mathrm{k}}\right\}$ is a set of shill bidder properties. Each property in $\mathrm{P}$ can be transformed into a piece of shilling-behavior evidence that can be used to identify and verify shills.

4. $\boldsymbol{\theta}=\left\{\theta_{a}, \theta_{b}, \ldots, \theta_{i},\right\}$ is a set of thresholds, where $\theta_{i}$ is the threshold for the basic mass assignment of evidence $i$, where $0 \leq \theta_{i} \leq 1$;

5. $\varphi$ is the threshold for the shilling scores of bidders, where $0 \leq \varphi \leq 1$. If a bidder's shilling score exceeds $\varphi$, the bidder's role in the auction system will be updated to "Shill."

In our abstract model, if a bidder's basic mass assignment for evidence $i$ exceeds $\theta_{i}$, the verification process would be triggered to validate the trustworthiness of the bidder. If all the basic mass assignments for a bidder are within the threshold, the role of the bidder in the auction system would be updated to "Trusted Bidder." On the other hand, if a verification process is performed on a bidder but the bidder's shilling score does not exceed $\varphi$, the bidder will be updated to "Suspect".

\subsection{Shilling behavior properties}

Our shill verification approach combines degrees of belief associated with individual properties of shilling behaviors. Such properties define evidence of actual shill behaviors. Here we elaborate on four types of evidence that corresponds to bidding properties considered in this paper.

Property A. Shill bidders typically avoid placing bids in a later stage of an auction so as to reduce the risk of winning. This evidence can be captured by considering the relative time at which bidders place their last bid. The earlier such a (last) bid is placed in 
an auction, the more suspicious is the bidder. We quantify this relative time of such a bid by Eq. (1).

$$
W_{\text {Shill }}^{P_{A}}=\frac{T_{\text {end }}-T_{\text {last }}}{T_{\text {duration }}}
$$

where $T_{\text {end }}$ is the ending time of the auction; $T_{\text {last }}$ is the

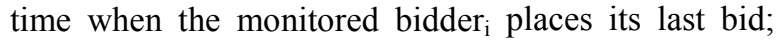
and $T_{\text {duration }}$ is the duration of the auction. Thus, in terms of this particular evidence in isolation, the likelihood of a bidder being a shill increases as $W_{\text {Shill }}^{P_{A}}$ increases.

Property B. Shill bidders have skewed bid distributions that are inclined toward particular sellers. The auction house maintains records of the number of bids a bidder has placed for every seller that the bidder has interacted with. Well-intentioned active bidders are expected to have placed bids in several different sellers' auctions, while shill bidders typically only deal with a very limited number of sellers, those they are colluding with. Thus shill suspect bidder $_{i}$ has a lower $\mathrm{BPS}_{(\mathrm{i}, \mathrm{j})}$ value for a specific seller $_{j}$ than other bidders, where $\mathrm{BPS}_{(\mathrm{i}, \mathrm{j})}$ gives the total number of bids that bidder $_{i}$ has placed on seller,'s auctions. We compute the proportion of bidder, ${ }_{i}$ s bids devoted to seller,'s auctions as follows:

$$
W_{\text {Shill }}^{P_{B}}=\frac{B P S_{(i, j)}}{\sum_{j=0, j \neq i}^{n} B P S_{(i, j)}}
$$

where $n$ is the total number of sellers that bidder $_{i}$ has interacted with.

Property C. Shill bidders generally win very few auctions. This property can be measured by Bids/Win (hereafter denoted as BPW). Shills should have a higher BPW score than normal bidders; however, considering that some shills may not win any auction, this will cause the denominator to be zero. Therefore, we use Wins/Bid (hereafter WPB) to capture this property, where WPB $=1 / \mathrm{BPW}$. The lower the WPB score, the more likely it is that the bidder is a shill. We define the winning frequency variation $\left(W_{\text {Shill }}^{P_{C}}\right)$ that distinguishes shill bidders from normal bidders as given by Eq. (3).

$$
W_{\text {Shill }}^{P_{c}}=1-W P B_{i}
$$

where $\mathrm{WPB}_{\mathrm{i}}$ is the wins per bid value of bidder $\mathrm{i}_{\mathrm{i}}$.

Property D. Shill bidders are not bargain hunters. Because a shill bidder's purpose is different from that of legitimate bidders, shill bidders typically do not favor items with lower prices. They may place bids in an auction that has a higher current bidding price rather than in some concurrent auctions that have lower current bidding prices. We have described how to apply model checking for this property in [1].

\subsection{The shill inference framework}

This section describes the basic idea of our shill inference approach. The framework for the shill bidder inference module is depicted in Figure 1.

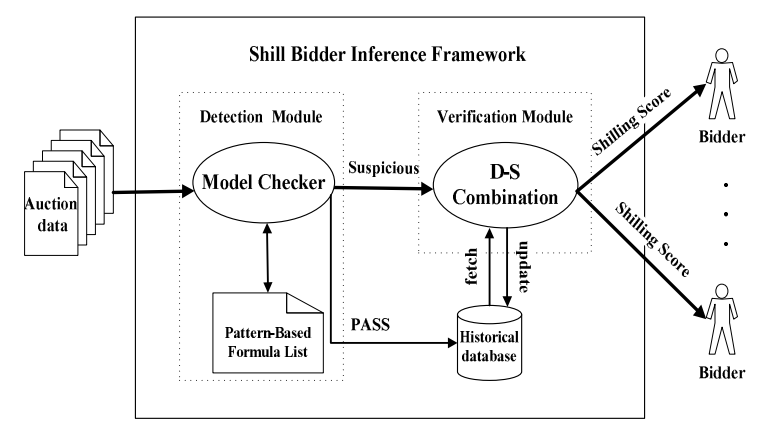

Figure 1. Shill bidder inference framework

The proposed shill inference approach is based on the following two key steps:

Step 1: Model checking based detection of suspicious shills. In this step, suspicious shill bidders in an auction are identified according to shill-bidding pattern-based temporal formulas. Such formulas (patterns of possible shilling behaviors) can drive a model-checker, as described in [1]. Further details for this step are beyond the scope of this paper.

Step 2: D-S Theory based shill verification. The shill suspects are verified mathematically in this step using a data fusion method that combines information from different aspects of bidders' behaviors. This verification process will further classify the shill suspects into categories reflecting the likelihood of an "actual" shill.

The shill inference procedure begins with the model checking procedure. Initially, every bidder in the auction house is categorized as a trusted bidder. When an auction ends, the deployed model checker performs a check on the bidders' bidding behaviors. Real-time auction data goes through the model checking module and each of the specified properties of shill behaviors is checked. The required statistical data for computing the basic mass assignments include the bidder's total number of bids in a certain period of time, the number of bids in the particular auction, the number of wins within the time period, the bids per seller ratio, the time of every bidder's last bid in the auction, and other details of the auction. Such data is saved in a historical database, and can be fetched when needed. The result for particular evidence from the model checking step is either "true" or "false," indicating if the shill property is satisfied or not satisfied by a shill suspect. If any "true" is returned, meaning any shilling behavior of a bidder is identified, 
the verification process for that bidder will be triggered after the model checking for all bidders are completed.

In the verification module, the separate pieces of evidence obtained from various behavioral properties in the model checking step are fused using D-S theory [8]. The result of the evidence combination process is a belief value that is used as a shilling score. Once the shilling score is calculated, the verification module updates each bidder's role according to the following rule:

$$
\forall b_{j} \in B:\left\{\begin{array}{c}
\forall p_{i} \in P: m_{i}\left(\text { shill }_{j}\right)<\theta_{i} \Rightarrow b_{j} \text { is a "Trusted Bidder" } \\
\exists p_{i} \in P: m_{i}\left(\text { shill }_{j}\right) \geq \theta_{i} \wedge \delta\left(\text { shill }_{i}\right)<\varphi \Rightarrow b_{j} \text { is a "Suspect" } \\
\delta\left(\text { shill }_{j}\right) \geq \varphi \quad \Rightarrow b_{j} \text { is a "Shill" }
\end{array}\right.
$$

where $m$ is a basic mass assignment function, to be discussed in Section 3.4, and $m_{\mathrm{i}}\left(\right.$ shill $\left._{\mathrm{j}}\right)$ represents the belief that $b_{\mathrm{j}}$ is a shill based on evidence factor $i$.

If a bidder's role changes, the new role will be committed to the database. If a bidder's role is labelled as "Shill," this bidder would likely be subject to further investigation and possible punishment, but this shill-handling step is outside the scope of this paper.

\subsection{Shill verification}

As mentioned earlier, the shill verification process employs a mathematical theory, Dempster-Shafer theory, to combine degrees of belief and provide a comprehensive result for shill detection. DempsterShafer theory is based on two ideas [13]: 1) obtaining degrees of belief for one question from subjective probabilities for a related question, and 2) Dempster's rule for combining such degrees of belief when they are based on independent items of evidence.

3.4.1. Basic mass assignment. For the shill detection problem in online auctions, the frame of discernment $\Theta$ (the set of all possible states) for any bidder is given by $\Theta=\{$ shill, $\neg$ shill $\}$. For this frame of discernment $\Theta$, the power set $2^{\Theta}$ has four elements, that is $2^{\Theta}=\{\varnothing$, $\{$ shill $\},\{\neg$ shill $\},\{$ either shill or not shill $\}\}$, where shill represents that the suspect is a shill bidder while $\neg$ shill means that the suspect is not a shill. Note that $\varnothing$ (empty set) means the suspect is neither a shill nor not a shill. The set \{either shill or not shill\} indicates that the detection system is uncertain if the suspect is or is not a shill. A mapping $m: 2^{\Theta} \rightarrow[0,1]$ assigns a mass to the subset of $\Theta$ and is called a basic mass assignment (BMA). $\mathrm{M}(\mathrm{A})$ represents the belief on the state of subset $A \subset \Theta$. In Dempster-Shafer theory, the mass of the empty set is zero and the basic mass assignment for each piece of evidence should satisfy the equation:

$$
1=\sum_{A \in 2^{\Theta}} m(A)
$$

In our case, this mean that $1=m($ shill $)+m(\neg$ shill $)+m(\mathrm{U})$, where $\mathrm{U}$ (uncertainty) represents the state of the subset \{either shill or not shill\}.

We can now provide basic mass assignments (BMA) for evidence factors $A, B, C$, and $D$, previously discussed. In our notations, $\alpha, \beta, \lambda$, and $\mu$ are the weights of evidence $A, B, C$, and $D$, respectively. A weight can be intuitively interpreted as the reliability of the associated evidence in the process of inferring online auction shills.

BMA for Evidence A: When a bidder is detected to have a tendency to stop bidding at an early stage, the bidder should be identified as a shill suspect. To capture evidence $A$, each bidder is assigned a mass in accordance with the time of its last bid. The mass assignment based on evidence $A$ is given by Eq. (4).

$$
\left.\begin{array}{l}
m_{A}\left(\operatorname{shill}_{i}\right)=\alpha * W_{\text {Shill }}^{P_{A}}=\alpha * \frac{T_{\text {end }}-T_{\text {last }}}{T_{\text {duration }}} \\
m_{A}\left(\neg \operatorname{shill}_{i}\right)=0 \\
m_{A}(U)=1-\alpha^{*} W_{\text {Shill }}^{P_{A}}=1-\alpha * \frac{T_{\text {end }}-T_{\text {last }}}{T_{\text {duration }}}
\end{array}\right\}
$$

BMA for Evidence B: When a bidder devotes most bids to a particular seller, the bidder should be suspected as a shill. The basic mass based on evidence $B$ is assigned to each bidder in the monitored auction to quantify the likelihood of the bidder being a shill. The basic mass assignment for evidence $B$ is given by Eq. (5).

$$
\left.\begin{array}{l}
m_{B}\left(\text { shill }_{i}\right)=\beta * W_{\text {Shill }}^{P_{B}}=\beta * \frac{B P S_{(i, j)}}{\sum_{j=0, j \neq i}^{n} B P S_{(i, j)}} \\
m_{B}\left(\neg \text { shill }_{i}\right)=0 \\
m_{B}(U)=1-\beta * W_{\text {Shill }}^{P_{B}}=1-\beta * \frac{B P S_{(i, j)}}{\sum_{j=0, j \neq i}^{n} B P S_{(i, j)}}
\end{array}\right\}
$$

BMA for Evidence C: When a bidder's winning ratio, in the form of wins per bid, is abnormal (e.g., lower than a threshold), the bidder should be suspected as a shill. The basic mass assignment that measures the winning ratio for each bidder is given by Eq. (6).

$$
\left.\begin{array}{l}
m_{C}\left(\text { shill }_{i}\right)=\lambda * W_{\text {Shill }}^{P_{C}}=\lambda *\left(1-W P B_{i}\right) \\
m_{C}\left(\neg \text { Shill }_{i}\right)=0 \\
m_{C}(U)=1-\lambda * W_{\text {Shill }}^{P_{C}}
\end{array}\right\}
$$

BMA for Evidence D: When a bidder passes up the chance to place a lower bid for an item in a concurrent auction, the bidder should be a shill suspect. Every 
bidder in the monitored auction is assigned a mass to indicate the belief that the bidder is a shill based on evidence $D$. For simplicity we let the unweighted mass be a constant $(x)$ for those bidders identified as a shill suspect by evidence $D$. For example, $x$ is set to 0.9 in our case study in Section 4. The value of $x$ should be predefined and used consistently in the process of inferring shills. To be meaningful, the value of $x$ should satisfy: $0.5 \leq x<1$. Eq. (7) provides the basic mass assignment for evidence $D$.

$$
\left.\begin{array}{l}
m_{D}\left(\text { shill }_{i}\right)=\mu x \\
m_{D}\left(\neg \text { shill }_{i}\right)=0 \\
m_{D}(U)=1-\mu x
\end{array}\right\}
$$

In each of the previous mass assignments, the mass assignment of the value zero for all $m_{x}\left(\neg\right.$ shill $\left.l_{i}\right)$ means that evidence $x$ gives zero degree of belief about the state of bidder ${ }_{i}$ being not a shill.

3.4.2. Evidence combination. Once the basic mass assignments are obtained, evidence is combined in a consistent manner to provide a more complete assessment of what the entire body of evidence implies. The evidence fusion procedure is carried out using Dempster's combination rule. The corresponding rule of combining evidence of shill in our context is derived as follows: belief $\left(\right.$ shill $\left._{i}\right)=m\left(\right.$ shill $\left._{i}\right)=m_{1}\left(\right.$ shill $\left._{i}\right) \oplus m_{2}\left(\right.$ shill $\left._{i}\right) \oplus \cdots \oplus m_{n}\left(\right.$ shill $\left._{i}\right)$ (8)

To combine multiple pieces of evidence from different properties of shill bidding behaviors, we can compute belief(shill $\left.\mathrm{l}_{\mathrm{i}}\right)$ by combining any pairs of evidence, then combining that result with the remaining third evidence, fourth evidence, and so on. The following rules combine evidences 1 and 2 :

$$
\begin{aligned}
& m_{1}\left(\operatorname{shill}_{i}\right) \oplus m_{2}\left(\operatorname{shill}_{i}\right)= \\
& \frac{1}{K}\left[m_{1}\left(\operatorname{shill}_{i}\right) m_{2}\left(\operatorname{shill}_{i}\right)+m_{1}\left(\operatorname{shill}_{i}\right) m_{2}(U)+m_{1}(U) m_{2}\left(\operatorname{shill}_{i}\right)\right] \\
& m_{1}\left(\neg \operatorname{shill}_{i}\right) \oplus m_{2}\left(\neg \operatorname{shill}_{i}\right)= \\
& \frac{1}{K}\left[m_{1}\left(\neg \operatorname{shill}_{i}\right) m_{2}\left(\neg \operatorname{shill}_{i}\right)+m_{1}\left(\neg \operatorname{shill}_{i}\right) m_{2}(U)+m_{1}(U) m_{2}\left(\neg \operatorname{shill}_{i}\right)\right] \\
& m_{1}(U) \oplus m_{2}(U)=\frac{1}{K} m_{1}(U) m_{2}(U), \text { where } \\
& K=m_{1}\left(\operatorname{shill}_{i}\right) m_{2}\left(\operatorname{shill}_{i}\right)+m_{1}\left(\operatorname{shill}_{i}\right) m_{2}(U)+ \\
& \left.\quad m_{1}(U) m_{2}\left(\operatorname{shill}_{i}\right)+m_{1}\left(\neg \operatorname{shill}_{i}\right) m_{2}(\neg \operatorname{shill})_{i}\right)+ \\
& \quad m_{1}\left(\neg \operatorname{shill}_{i}\right) m_{2}(U)+m_{1}(U) m_{2}\left(\neg \operatorname{shill}_{i}\right)+m_{1}(U) m_{2}(U)
\end{aligned}
$$

Because belief $\left(\neg \operatorname{shill}_{i}\right)=m\left(\neg \operatorname{shill}_{i}\right)$ and $\mathrm{m}\left(\neg \operatorname{shill}_{i}\right)=0$, we have belief $\left(\neg \operatorname{shill} l_{i}\right)=0$. After calculating the belief value for bidder $b_{\mathrm{i}}$, the value of belief $\left(\right.$ shill $\left._{\mathrm{i}}\right)$ is assigned to $b_{\mathrm{i}}$ as a shilling score to indicate the degree of credibility of $b_{\mathrm{i}}$.

\section{Case study and analysis results}

\subsection{Data collection and processing}

The data used in our case study was collected from recent auctions on eBay with the title "Microsoft Xbox 360 Complete System \& 20G Hard Drive." We investigated the bidding history of bidders that participated in this auction, counting each bidder's number of wins, total number of bids, number of bids in this particular auction, sorting the bidders in the reverse order of the time they placed their last bid after the auction began, etc. All the required information can be obtained from eBay's website.

The statistical results are shown in Table 1. Since eBay only provides the number of total bids and the number of items a bidder has placed bids in the last 30 days, we manually count the number of wins in the last 30 days in order to get a consistent numerical result. We assigned the (unweighted) basic mass for evidence $D$ as 0.9 rather than 1 , to take into account the uncertain factors involved in evidence $D$ indicating actual shill behavior. While the value of 0.9 is somewhat arbitrary, we set it relatively high since we do believe that bidding a higher amount in concurrent auctions is a strong shill indicator ${ }^{1}$. In this auction, 8 out of the total 13 bidders $(61.5 \%)$ who placed bids in the auction also placed bids in a higher priced concurrent auction that is held by the same seller for the same auctioned item. All of these 8 bidders are shill suspects, but not all of them are verified as shills, as we will see in the verification results in Section 4.2.

The basic masses assigned for evidence $A, B, C$, and $D$ according to eq. (4), (5), (6), and (7) are shown in Table2. The $\alpha, \beta, \lambda$, and $\mu$ weights of evidence were set as $.8, .95, .7$ and .8 , respectively.

The belief of shilling behavior and the uncertainty of shilling behavior calculated using eq. (8), (9), (10), (11) and (12) are shown in Table 2. In the formulas, the masses of $m_{\mathrm{A}}\left(\neg \operatorname{shill}_{i}\right), m_{\mathrm{B}}\left(\neg \operatorname{shill}_{i}\right), m_{\mathrm{C}}\left(\neg \operatorname{shill}_{i}\right)$, and $m_{\mathrm{D}}\left(\neg \operatorname{shill}_{i}\right)$ are all by default 0 and are not shown in Table 2. The last column of the table shows the updated role of each bidder after the inference process is completed. These roles are assigned in accordance with the following rule:

\section{$\left(m_{A}\left(\right.\right.$ shill $\left._{i}\right) \leq 0.5 \& m_{B}\left(\right.$ shill $\left._{i}\right) \leq 0.5 \& m_{(}\left(\right.$shill $\left._{i}\right) \leq 0.7 \& m_{D}\left(\right.$ shill $\left._{i}\right) \leq 0.5 \Rightarrow$ ThstedBidder $\left\{\left(m_{A}\left(\right.\right.\right.$ shill $\left._{i}\right)>0.5 \| m_{B}\left(\right.$ shill $\left._{i}\right)>0.5 \| m_{(}\left(\right.$shill $\left._{i}\right)>0.7 \| m_{\supset}\left(\right.$ shill $\left.\left._{i}\right)>0.5\right) \&\left(\delta\right.$ shill $\left.\left._{i}\right)<0.99\right) \Rightarrow$ Sispect (dshill $) \geq 0.99 \Rightarrow$ Shill}

In this example, we set $\theta=\{0.5,0.5,0.7,0.5\}$ and $\varphi=0.99$. To make the thresholds meaningful and practical in identifying suspicious bidders, it is

1 In actual deployment, an appropriate value for evidence $\mathrm{D}$ can be learned through training auction data to achieve precise results. 
reasonable that the values of the thresholds should be greater than or equal to 0.5 . Note that $\theta_{c}$ is assigned 0.7 because statistically, even the win-ratio of an ordinary, trusted bidder is typically less than 0.5 . Hence $\theta_{c}$ should be higher than 0.5 . To reduce the number of false positives generated in our proposed approach, the shilling score threshold $\varphi$ should be sufficiently high. In Table 2, those numerical values that exceed their respective threshold are shown as double underlined. Note that we consider that false negatives that could possibly be generated in our approach are of equal importance as false positives; however it is beyond the scope of this paper.

\subsection{Analysis results}

Trusted Bidder: In this case, we show what kind of bidder is considered innocent. Considering bidders $\mathrm{t}^{* * *} \mathrm{o}(8), \mathrm{u}^{* * *} \mathrm{l}(107)$, and $\mathrm{c}^{* * *} \mathrm{~d}(40)$, none of their degrees of belief for evidence A, B, C, or D exceeds $\theta$. Therefore, the three bidders should pass all checks using the model checking method and they are automatically updated to the status of "Trusted Bidder."

Suspect: In this case, we show that a bidder is initially suspected as a shill, but later verified as not a shill. Consider bidder $\mathrm{e}^{* * * 1(13)}$ in Table 1. Bidder

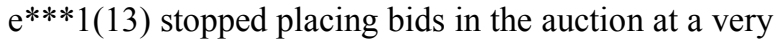
early time and devoted more than half (54\%) of its total bids to this seller's auction. Such evidence leads the system to suspect that $\mathrm{e}^{* * * 1} 1(13)$ is a shill. The

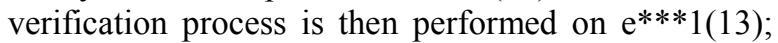
however, the result shows that the shilling score for $\mathrm{e}^{* * * 1} 1(13)$ is only 0.9152 , which does not reach the shill threshold (0.99). Therefore, bidder $\mathrm{e}^{* * * 1(13)}$ is verified as not a shill at this moment, although $\mathrm{e}^{* * *(13)}$ is still a suspect according to the role assignment rule in Section 4.1.

Table 1. Auction data after processing

\begin{tabular}{|c|c|c|c|c|c|c|}
\hline Bidder & $\begin{array}{l}\text { \# of bids in this } \\
\text { auction }\end{array}$ & total bids* & $\begin{array}{c}\text { \# of items bid } \\
\text { on }\end{array}$ & $\begin{array}{l}\text { bid activity with } \\
\text { this seller ** }\end{array}$ & \# of wins & $\begin{array}{l}\text { time from last bid to } \\
\text { the end of } \\
\text { auction*** }\end{array}$ \\
\hline $4 * * * 4(5)$ & 14 & 17 & 3 & $82 \%$ & 0 & 342 \\
\hline $\mathrm{b}^{* * *} 0(15)$ & 5 & 9 & 4 & $56 \%$ & 3 & 411 \\
\hline $\mathrm{b}^{* * *} \mathrm{a}(67)$ & 1 & 115 & 50 & $2 \%$ & 60 & 3079 \\
\hline$u^{* * *} 1(107)$ & 1 & 44 & 20 & $2 \%$ & 12 & 3551 \\
\hline $\mathrm{t}^{* * *} \mathrm{o}(8)$ & 1 & 160 & 95 & $1 \%$ & 8 & 4031 \\
\hline $\mathrm{c}^{* * *} \mathrm{~d}(40)$ & 1 & 5 & 4 & $20 \%$ & 2 & 4470 \\
\hline $\mathrm{o}^{* * *} \mathrm{O}(0)$ & 3 & 23 & 10 & $60 \%$ & 0 & 5389 \\
\hline $\mathrm{o}^{* * * \mathrm{u}} \mathrm{u}(0)$ & 3 & 27 & 12 & $70 \%$ & 0 & 12863 \\
\hline $2 * * * 0(20)$ & 2 & 68 & 21 & $10 \%$ & 20 & 18973 \\
\hline $\mathrm{y}^{* * * 3(0)}$ & 2 & 27 & 11 & $25 \%$ & 0 & 51615 \\
\hline$v^{* * * 8(0)}$ & 2 & 5 & 2 & $100 \%$ & 0 & 58923 \\
\hline $6 * * * 0(50)$ & 4 & 55 & 32 & $7 \%$ & 12 & 70095 \\
\hline $\mathrm{e}^{* * *} 1(13)$ & 2 & 11 & 7 & $54 \%$ & 8 & 83830 \\
\hline \multicolumn{7}{|c|}{$\begin{array}{l}* \text { eBay only provides a bidder's bid history for the last } 30 \text { days. } \\
* * \text { This shows the percentage of all bids from this bidder that went to this specific seller. } \\
* * * \text { time from last bid to the end of auction (in seconds) = The duration of the auction - duration of a bidder's last bid since the auction } \\
\text { begins (Oct-21-08 14:00:00). }\end{array}$} \\
\hline
\end{tabular}

Shill: In this case, we show how shills can be detected and then verified. Take the case of checking and verifying $\mathrm{v}^{* * * 8(0)}$ as an example. In Table 2, the degrees of belief that $\mathrm{v}^{* * * 8(0)}$ is a shill from evidence $\mathrm{A}, \mathrm{B}, \mathrm{C}$, and $\mathrm{D}$ are $0.5456,0.95,0.7$ and 0.72, respectively. Since all four values exceed their respective thresholds, the verification process must be activated to validate $\mathrm{v}^{* * *} 8(0)$ 's status. Given Eq. (8), (9), (10), (11) and (12), we fused the degrees of belief from all available evidence to obtain the ultimate belief of shill, which is also the shilling score. The shilling score for $\mathrm{v}^{* * * 8} 8(0)$ is 0.9981 , which is greater than $\varphi(0.99)$. Based on the verification result, we believe $\mathrm{v}^{* * * 8(0)}$ to be a shill; thus we update the role of $\mathrm{v}^{* * * 8(0)}$ to "Shill." There are reasons to consider this ultimate result as reasonable. First, $\mathrm{v}^{* * * 8(0)}$ might see that the price in this auction was lower than that of a concurrent auction and that this auction would end earlier than the other one, but yet this bidder still placed bids on the other auction. Second, $\mathrm{v}^{* * * 8(0)}$ placed 5 bids but did not win any auction. Third, $\mathrm{v}^{* * * 8(0)}$ placed bids consecutively at very early times. Fourth, $\mathrm{v}^{* * * 8(0)}$ did not place any other bids later in the auction. Finally, the bidder $\mathrm{v}^{* * * 8(0)}$ devoted all bids to the seller. So, the verification result is consistent with the manually observed result. 
Table 2. The basic mass assignments and shill verification results

\begin{tabular}{|c|c|c|c|c|c|c|c|c|c|c|c|}
\hline Bidder & $\mathrm{m}_{\mathrm{A}}\left(\right.$ shill $\left._{\mathrm{i}}\right)$ & $\mathrm{m}_{\mathrm{A}}(\mathrm{U})$ & $\mathrm{m}_{\mathrm{B}}\left(\right.$ shill $\left._{i}\right)$ & $\mathrm{m}_{\mathrm{B}}(\mathrm{U})$ & $\mathrm{m}_{\mathrm{C}}\left(\operatorname{shill}_{i}\right)$ & $\mathrm{m}_{\mathrm{C}}(\mathrm{U})$ & $\mathrm{mp}_{\mathrm{D}}\left(\right.$ shill $\left._{i}\right)$ & $\mathrm{m}_{\mathrm{D}}(\mathrm{U})$ & \begin{tabular}{|c|} 
Belief of Shill(Shill \\
Score)
\end{tabular} & $\begin{array}{c}\text { Uncertainty of } \\
\text { Shill }\end{array}$ & Result \\
\hline $4^{* * * 4(5)}$ & 0.0032 & 0.9968 & $\underline{\underline{0.779}}$ & 0.221 & $\underline{\underline{07}}$ & 0.3 & $\underline{\underline{0.72}}$ & 0.2800 & 0.9815 & 0.0185 & Suspect \\
\hline $\mathrm{b}^{* * * *} 0(15)$ & 0.0038 & 0.9962 & $\underline{\underline{0.504}}$ & 0.496 & 0.4669 & 0.5331 & $\underline{0.72}$ & 0.2800 & 0.9262 & 0.0738 & Suspect \\
\hline$b^{* * * * a(67)}$ & 0.0285 & 0.9715 & 0.019 & 0.981 & 0.3346 & 0.6654 & $\underline{\underline{0.72}}$ & 0.2800 & 0.8224 & 0.1776 & Suspect \\
\hline $\mathrm{u}^{* * * 1}(107)$ & 0.0329 & 0.9671 & 0.019 & 0.981 & 0.5089 & 0.4911 & 0.00 & 1.0000 & 0.5341 & 0.4659 & Trusted Bidder \\
\hline $\mathrm{t}^{* * *} \mathrm{o}(8)$ & 0.0373 & 0.9627 & 0.0095 & 0.9905 & 0.665 & 0.335 & 0.00 & 1.0000 & 0.6806 & 0.3194 & Trusted Bidder \\
\hline $\mathrm{c}^{* * *} \mathrm{~d}(40)$ & 0.0414 & 0.9586 & 0.19 & 0.81 & 0.42 & 0.58 & 0.00 & 1.0000 & 0.5496 & 0.4504 & Trusted Bidder \\
\hline $\mathrm{o}^{* * * *} \mathrm{o}(0)$ & 0.0499 & 0.9501 & $\underline{\underline{0.57}}$ & 0.43 & $\underline{\underline{07}}$ & 0.3 & 0.00 & 1.0000 & 0.8774 & 0.1226 & Suspect \\
\hline $0^{* * *} \mathrm{u}(0)$ & 0.1191 & 0.8809 & $\underline{\underline{0.665}}$ & 0.335 & $\underline{\underline{0.7}}$ & 0.3 & $\underline{\underline{0.72}}$ & 0.2800 & 0.9752 & 0.0248 & Suspect \\
\hline $2 * * * 0(20)$ & 0.1757 & 0.8243 & 0.095 & 0.905 & 0.4941968 & 0.5058032 & $\underline{\underline{0.72}}$ & 0.2800 & 0.8943 & 0.1057 & Suspect \\
\hline $\mathrm{y}^{* * *} 3(0)$ & 0.4779 & 0.5221 & 0.2375 & 0.7625 & $\underline{\underline{0.7}}$ & 0.3 & $\underline{\underline{0.72}}$ & 0.2800 & 0.9666 & 0.0334 & Suspect \\
\hline$r^{* * *} 8(0)$ & $\underline{\underline{0.5456}}$ & 0.4544 & $\underline{\underline{0.95}}$ & 0.05 & $\underline{\underline{07}}$ & 0.3 & $\underline{\underline{0.72}}$ & 0.2800 & $\underline{\underline{0.9981}}$ & 0.0019 & Shill \\
\hline $6 * * * 0(50)$ & $\underline{\underline{0.6490}}$ & 0.3510 & 0.0665 & 0.9335 & 0.5474 & 0.4526 & $\underline{\underline{0.72}}$ & 0.2800 & 0.9585 & 0.0415 & Suspect \\
\hline$e^{* * * 1(13)}$ & $\underline{\underline{0.7847}}$ & 0.2153 & $\underline{\underline{0.513}}$ & 0.487 & 0.1911 & 0.8089 & 0.00 & 1.0000 & 0.9152 & 0.0848 & Suspect \\
\hline
\end{tabular}

\section{Conclusions and future work}

In this paper, we introduced a two-step shill detection and verification approach: 1) detecting shill suspects using model checking technique, and 2) verifying shills using Dempster-Shafer theory. The first step of our approach can potentially support detection of shill suspects in real-time and the second step combines multiple pieces of evidence and provides a more accurate result than the one based on any single piece of evidence. The case study shows that our proposed approach can be valid and applicable in real world deployment.

In our future research, we will experiment with integrating more pieces of evidence that indicate both shills and not shills. We believe that the DempsterShafer theory of evidence approach, as a theoretically generalized Bayesian inference method, can provide a practical approach and enhance system performance for shill verification in online auctions.

\section{References}

[1] H. Xu and Y. T. Cheng, "Model Checking Bidding Behaviors in Internet Concurrent Auctions," International Journal of Computer Systems Science \& Engineering, Vol. 22, No. 4, 2007, pp. 179-191.

[2] H. Xu, S. M. Shatz, and C. K. Bates, "A Framework for Agent-Based Trust Management in Online
Auctions," In Proceedings of the 5th International Conference on Information Technology: New Generations (ITNG 2008), Las Vegas, April 2008, pp. 149-155.

[3] R. J. Kauffman and C. A. Wood, "Running up the Bid: Detecting, Predicting, and Preventing Reserve Price Shilling in Online Auctions," In Proceedings of the 5th International Conference on Electronic Commerce, Pittsburgh, October 2003, pp. 259-265.

[4] J. Trevathan and W. Read, "Detecting Shill Bidding in Online English Auctions," Handbook of Research on Social and Organizational Liabilities in Information Security, M. Gupta and R. Sharman (eds.), Information Science Reference, 2009, pp. 446-470.

[5] D. H. Chau, S. Pandit, and C. Faloutsos, "Detecting Fraudulent Personalities in Networks of Online Auctioneers," In Proceedings of the 10th European Conference on Principles and Practice of Knowledge Discovery in Database (PKDD 2006), 2006, pp. 103114.

[6] T.M. Chen, and V. Venkataramanan, "DempsterShafer Theory for Intrusion Detection in Ad Hoc Networks," In Proceedings of the IEEE Internet Computing, 2005, pp. 35-41.

[7] S. Panigrahi, A. Kundu, S. Sural, and A.K. Majunmdar, "Use of Dempster-Shafer Theory and Bayesian Inferencing for Fraud Detection in Mobile Communication Networks," Lecture Notes in Computer Science, Spring Berlin /Heidelberg, Vol. 4586, 2007, pp. 446-460.

[8] G. Shafer, A Mathematical Theory of Evidence, Princeton University press, 1976. 